\title{
Permanence for a modified Leslie-Gower predator-prey model with Beddington-DeAngelis functional response and feedback controls
}

Qin Yue*

"Correspondence:

xhper1978@sina.com

College of Finance and

Mathematics, West Anhui University,

Liuan, Anhui 237000, P.R. China

\begin{abstract}
A modified Leslie-Gower predator-prey system with Beddington-DeAngelis functional response and feedback controls is studied. By applying the differential inequality theory, sufficient conditions which guarantee the permanence of the system are obtained. Our results improve the main results of Zhang et al. (Abstr. Appl. Anal. 2014:252579, 2014). One example is presented to verify our main results.
\end{abstract}

Keywords: permanence; Leslie-Gower model; Beddington-DeAngelis functional response; feedback controls

\section{Introduction}

Let $f(t)$ be a continuous bounded function on $\mathbb{R}$, and we set

$$
f^{l}=\inf _{t \in \mathbb{R}} f(t), \quad f^{u}=\sup _{t \in \mathbb{R}} f(t)
$$

Leslie $[1,2]$ introduced the following two species Leslie-Gower predator-prey model:

$$
\left\{\begin{array}{l}
\dot{x}(t)=\left(r_{1}-b_{1} x(t)\right) x(t)-p(x(t)) y(t), \\
\dot{y}(t)=\left(r_{2}-a_{2} \frac{y(t)}{x(t)}\right) y(t)
\end{array}\right.
$$

where $x(t), y(t)$ stand for the population (the density) of the prey and the predator at time $t$, respectively. The parameters $r_{1}$ and $r_{2}$ are the intrinsic growth rates of the prey and the predator, respectively. $b_{1}$ measures the strength of competition among individuals of species $x$. The value $\frac{r_{1}}{b_{1}}$ is the carrying capacity of the prey in the absence of predation. The predator consumes the prey according to the functional response $p(x)$ and grows logistically with growth rate $r_{2}$ and carrying capacity $\frac{r_{2} x}{a_{2}}$ proportional to the population size of the prey (or prey abundance). The parameter $a_{2}$ is a measure of the food quantity that the prey provides and converted to predator birth. The term $y / x$ is the Leslie-Gower term which measures the loss in the predator population due to rarity (per capita $y / x$ ) of its favorite food. Leslie model is a predator-prey model where the carrying capacity of the 
predator is proportional to the number of prey, stressing the fact that there are upper limits to the rates of increase in both prey $x$ and predator $y$, which are not recognized in the Lotka-Volterra model.

The main shortcoming of (1.1) is that in the case of severe scarcity, $y$ can switch over to other populations but its growth will be limited by the fact that its most favorite food $x$ is not available in abundance. In order to overcome this shortcoming, recently, AzizAlaoui and Daher Okiye [3] suggested to add a positive constant $d$ to the denominator and proposed the following predator-prey model with modified Leslie-Gower and Hollingtype II schemes:

$$
\left\{\begin{array}{l}
\dot{x}(t)=\left(r_{1}-b_{1} x(t)-\frac{a_{1} y(t)}{x(t)+k_{1}}\right) x(t), \\
\dot{y}(t)=\left(r_{2}-\frac{a_{2} y(t)}{x(t)+k_{2}}\right) y(t),
\end{array}\right.
$$

where $r_{1}, b_{1}, r_{2}, a_{2}$ have the same meaning as in models (1.1). $a_{1}$ is the maximum value of the per capita reduction rate of $x$ due to $y, k_{1}$ (respectively, $k_{2}$ ) measures the extent to which the environment provides protection to prey $x$ (respectively, to the predator $y$ ). They obtained the boundedness and global stability of positive equilibrium of system (1.2). Yu [4] studied the structure, linearized stability and the global asymptotic stability of equilibria of (1.2). Zhu and Wang [5] obtained sufficient conditions for the existence and global attractivity of positive periodic solutions of system (1.2) with periodic coefficients. Yu and Chen [6] further considered the permanence and existence of a unique globally attractive positive almost periodic solution of system (1.2) with almost periodic coefficients and mutual interference. Considering that Beddington-DeAngelis functional response preformed better than Holling-type II functional response, $\mathrm{Yu}$ [7] incorporated the Beddington-DeAngelis functional response into system (1.2) and considered the following model which is the generalization of model (1.2):

$$
\left\{\begin{array}{l}
\dot{x}(t)=\left(r_{1}-b_{1} x(t)-\frac{a_{1} y(t)}{\alpha+\beta x(t)+\gamma y(t)}\right) x(t), \\
\dot{y}(t)=\left(r_{2}-\frac{a_{2}(y(t)}{x(t)+k_{2}}\right) y(t) .
\end{array}\right.
$$

Sufficient conditions on the global asymptotic stability of a positive equilibrium were obtained by Yu [7]. Pal and Mandal [8] considered the Hopf bifurcation of system (1.3) with strong Allee effect. Zhang [9] studied the permanence and existence of an almost periodic solution of system (1.3) with almost periodic coefficients.

Based on Zhang [9], Zhang et al. [10] incorporated the feedback control into model (1.3) with almost periodic coefficients and considered the following model:

$$
\left\{\begin{array}{l}
\dot{x}(t)=x(t)\left(a_{1}(t)-b(t) x(t)-\frac{c(t) y(t)}{\alpha(t)+\beta(t) x(t)+\gamma(t) y(t)}-e_{1}(t) u(t)\right) \\
\dot{y}(t)=y(t)\left(a_{2}(t)-\frac{r(t) y(t)}{x(t)+k(t)}-e_{2}(t) v(t)\right) \\
\dot{u}(t)=-d_{1}(t) u(t)+p_{1}(t) x(t-\tau) \\
\dot{v}(t)=-d_{2}(t) v(t)+p_{2}(t) y(t-\tau)
\end{array}\right.
$$

where $b(t), c(t), r(t), k(t), \alpha(t), \beta(t), \gamma(t), a_{i}(t), d_{i}(t), p_{i}(t)$ and $e_{i}(t)(i=1,2)$ are all continuous, almost periodic functions and satisfy

$$
\begin{aligned}
& \min _{i=1,2}\left\{b^{l}, c^{l}, r^{l}, k^{l}, \alpha^{l}, \beta^{l}, \gamma^{l}, a_{i}^{l}, d_{i}^{l}, p_{i}^{l}, e_{i}^{l}\right\}>0, \\
& \max _{i=1,2}\left\{b^{u}, c^{u}, r^{u}, k^{u}, \alpha^{u}, \beta^{u}, \gamma^{u}, a_{i}^{u}, d_{i}^{u}, p_{i}^{u}, e_{i}^{u}\right\}<+\infty .
\end{aligned}
$$


Set

$$
M_{1}=\frac{a_{1}^{u}}{b^{l}}, \quad M_{2}=\frac{a_{2}^{u}\left(M_{1}+k^{u}\right)}{r^{l}}, \quad L_{1}=\frac{p_{1}^{u} M_{1}}{d_{1}^{l}}, \quad L_{2}=\frac{p_{2}^{u} M_{2}}{d_{2}^{l}} .
$$

Suppose that system (1.4) holds together with the following initial conditions:

$$
\begin{array}{ll}
x(s)=\phi(s) \geq 0, \quad s \in[-\tau, 0], & \phi(0)>0, \\
y(s)=\varphi(s) \geq 0, \quad s \in[-\tau, 0], & \varphi(0)>0, \\
u(0)>0, \quad v(0)>0 . &
\end{array}
$$

By using the comparison theorem of differential equation, Zhang et al. [10] obtained the following result.

Theorem A ([10]) Assume that

$$
\left(\mathrm{H}_{1}\right) \quad a_{1}^{l}-\frac{c^{u}}{\gamma^{l}}-e_{1}^{u} L_{1}>0 \quad \text { and } \quad a_{2}^{l}-e_{2}^{u} L_{2}>0
$$

hold, then system (1.4) with initial conditions (1.6) is permanent, i.e., any positive solution $(x(t), y(t), u(t), v(t))^{T}$ of system (1.4) with initial conditions (1.6) satisfies

$$
\begin{aligned}
& m_{1} \leq \liminf _{t \rightarrow+\infty} x(t) \leq \limsup _{t \rightarrow+\infty} x(t) \leq M_{1}, \quad m_{2} \leq \liminf _{t \rightarrow+\infty} y(t) \leq \limsup _{t \rightarrow+\infty} y(t) \leq M_{2}, \\
& l_{1} \leq \liminf _{t \rightarrow+\infty} u(t) \leq \limsup _{t \rightarrow+\infty} y(t) \leq L_{1}, \quad l_{2} \leq \liminf _{t \rightarrow+\infty} v(t) \leq \limsup _{t \rightarrow+\infty} y(t) \leq L_{2},
\end{aligned}
$$

where $m_{i}, l_{i}, M_{i}$ and $L_{i}(i=1,2)$ are positive constants.

Theorem A shows that feedback control variables play important roles in the persistent property of system (1.4). But the question is whether or not the feedback control variables have influence on the permanence of the system. Many papers (see [11-13] and the references cited therein) have showed that feedback control variables have no influence on the permanent property of continuous system with feedback control. Thus, in this paper, we will apply the analysis technique of Chen et al. [11] to establish sufficient conditions, which is independent of feedback control variables, to ensure the permanence of the system. In fact, we obtain the following main result.

\section{Theorem B Assume that}

$$
\left(\mathrm{H}_{2}\right) \quad a_{1}^{l}-\frac{c^{u}}{\gamma^{l}}>0
$$

holds, then system (1.4) with initial conditions (1.6) is permanent.

Comparing with Theorem A, it is easy to see that $\left(\mathrm{H}_{2}\right)$ in Theorem $B$ is weaker than $\left(\mathrm{H}_{1}\right)$ in Theorem $\mathrm{A}$, and feedback control variables have no influence on the permanent property of system (1.4), so our results improve the main results in Zhang et al. [10]. 
The organization of this paper is as follows. In Section 2, we introduce several lemmas, and the permanence of system (1.4) is then studied in this section. In Section 3, a suitable example together with its numerical simulations is given to illustrate the feasibility of the main results.

\section{Permanence}

Now let us state several lemmas which will be useful in proving the main results of this section.

Lemma 2.1 ([14]) If $a>0, b>0$ and $\dot{x} \geq x(b-a x)$, when $t \geq 0$ and $x(0)>0$, we have

$$
\liminf _{t \rightarrow+\infty} x(t) \geq \frac{b}{a}
$$

If $a>0, b>0$ and $\dot{x} \leq x(b-a x)$, when $t \geq 0$ and $x(0)>0$, we have

$$
\limsup _{t \rightarrow+\infty} x(t) \leq \frac{b}{a}
$$

Lemma 2.2 ([11]) Assume that $a>0, b(t)>0$ is a bounded continuous function and $x(0)>0$. Further suppose that:

(i)

$$
\dot{x}(t) \leq-a x(t)+b(t),
$$

then for all $t \geq s$,

$$
x(t) \leq x(t-s) \exp \{-a s\}+\int_{t-s}^{t} b(\tau) \exp \{a(\tau-t)\} d \tau .
$$

Especially, if $b(t)$ is bounded above with respect to $M$, then

$$
\limsup _{t \rightarrow+\infty} x(t) \leq \frac{M}{a} .
$$

(ii)

$$
\dot{x}(t) \geq-a x(t)+b(t),
$$

then for all $t \geq s$,

$$
x(t) \geq x(t-s) \exp \{-a s\}+\int_{t-s}^{t} b(\tau) \exp \{a(\tau-t)\} d \tau .
$$

Especially, if $b(t)$ is bounded above with respect to $m$, then

$$
\liminf _{t \rightarrow+\infty} x(t) \geq \frac{m}{a} .
$$

The following lemma is a direct conclusion of [10]. 
Lemma 2.3 For any positive solution $(x(t), y(t), u(t), v(t))^{T}$ of system (1.4) with initial conditions (1.6), there exist four positive constants $M_{i}$ and $L_{i}(i=1,2)$ such that

$$
\begin{aligned}
& \limsup _{t \rightarrow+\infty} x(t) \leq M_{1}, \quad \limsup _{t \rightarrow+\infty} y(t) \leq M_{2}, \\
& \limsup _{t \rightarrow+\infty} u(t) \leq L_{1}, \quad \limsup _{t \rightarrow+\infty} v(t) \leq L_{2},
\end{aligned}
$$

where $M_{i}$ and $L_{i}(i=1,2)$ are defined in (1.5).

Lemma 2.4 Suppose that $\left(\mathrm{H}_{2}\right)$ holds, then there exist two positive constants $m_{1}$ and $l_{1}$ such that any positive solution $(x(t), y(t), u(t), v(t))^{T}$ of system (1.4) with initial conditions (1.6) satisfies

$$
\liminf _{t \rightarrow+\infty} x(t) \geq m_{1}, \quad \liminf _{t \rightarrow+\infty} u(t) \geq l_{1}
$$

where $m_{1}$ and $l_{1}$ are defined in the proof.

Proof According to Lemma 2.3, there exists enough large $T_{1}>0$ such that for $t \geq T_{1}$,

$$
x(t) \leq 2 M_{1}, \quad u(t) \leq 2 L_{1} .
$$

Thus, it follows from (2.2) and the first equation of system (1.4) that

$$
\begin{aligned}
\dot{x}(t) & =x(t)\left(a_{1}(t)-b(t) x(t)-\frac{c(t) y(t)}{\alpha(t)+\beta(t) x(t)+\gamma(t) y(t)}-e_{1}(t) u(t)\right) \\
& \geq x(t)\left(a_{1}^{l}-2 b^{u} M_{1}-\frac{c^{u}}{\gamma^{l}}-2 e_{1}^{u} L_{1}\right) \\
& \triangleq Q x(t),
\end{aligned}
$$

where $Q=a_{1}^{l}-2 b^{u} M_{1}-\frac{c^{u}}{\gamma^{l}}-2 e_{1}^{u} L_{1}<a_{1}^{l}-2 b^{u} M_{1}<a_{1}^{u}-2 b^{b} \frac{a_{1}^{u}}{b^{l}}=-a_{1}^{u}<0$.

Integrating both sides of (2.3) from $\eta(\eta \leq t)$ to $t$ leads to

$$
\frac{x(t)}{x(\eta)} \geq \exp \{Q(t-\eta)\}
$$

or

$$
x(\eta) \leq x(t) \exp \{-Q(t-\eta)\}
$$

Particularly, take $\eta=t-\tau$, one can get

$$
x(t-\tau) \leq x(t) \exp \{-Q \tau\} .
$$

Substituting (2.5) into the third equation of system (1.4) leads to

$$
\dot{u}(t) \leq-d_{1}^{l} u(t)+p_{1}^{u} x(t) \exp \{-Q \tau\} .
$$


Applying Lemma 2.2(i) to the above differential inequality, for $0 \leq s \leq t$, one has

$$
\begin{aligned}
u(t) \leq u(t-s) \exp \left\{-d_{1}^{l} s\right\}+\int_{t-s}^{t} p_{1}^{u} x(\eta) \exp \{-Q \tau\} \exp \left\{d_{1}^{l}(\eta-t)\right\} d \eta \\
\underset{\operatorname{from}(2.4)}{\leq} u(t-s) \exp \left\{-d_{1}^{l} s\right\} \\
\quad+\int_{t-s}^{t} p_{1}^{u} x(t) \exp \{-Q(t-\eta)\} \exp \{-Q \tau\} \exp \left\{d_{1}^{l}(\eta-t)\right\} d \eta \\
\leq u(t-s) \exp \left\{-d_{1}^{l} s\right\}+p_{1}^{u} x(t) \frac{1}{Q}(1-\exp \{-Q s\}) \exp \{-Q \tau\}
\end{aligned}
$$

where we have used the fact that $\max _{\eta \in[t-s, t]} \exp \left\{d_{1}^{l}(\eta-t)\right\}=\exp \{0\}=1$.

Note that there exists $K$ such that $2 e_{1}^{u} L_{1} \exp \left\{-d_{1}^{l} K\right\}<\frac{\beta}{2}$, as $s \geq K$, where $\beta=a_{1}^{l}-\frac{c^{u}}{\gamma^{l}}>0$ according to $\left(\mathrm{H}_{2}\right)$. In fact, we can choose $K>\frac{1}{d_{1}^{l}} \ln \frac{4 e_{1}^{u} L_{1}}{\beta}$. And so, fix $K$, combined with (2.7), we can obtain

$$
\begin{aligned}
u(t) & \leq u(t-K) \exp \left\{-d_{1}^{l} K\right\}+p_{1}^{u} x(t) \frac{1}{Q}(1-\exp \{-Q K\}) \exp \{-Q \tau\} \\
& \leq 2 L_{1} \exp \left\{-d_{1}^{l} K\right\}+p_{1}^{u} x(t) \frac{1}{Q}(1-\exp \{-Q K\}) \exp \{-Q \tau\} \\
& \leq 2 L_{1} \exp \left\{-d_{1}^{l} K\right\}+D x(t)
\end{aligned}
$$

for all $t>T_{1}+K$, where $D=p_{1}^{u} \frac{1}{Q}(1-\exp \{-Q K\}) \exp \{-Q \tau\}>0$.

Substituting (2.8) into the first equation of system (1.4), for all $t>T_{1}+K$, one has

$$
\begin{aligned}
\dot{x}(t) & \geq x(t)\left(a_{1}^{l}-b^{u} x(t)-\frac{c^{u}}{\gamma^{l}}-2 e_{1}^{u} L_{1} \exp \left\{-d_{1}^{l} K\right\}-e_{1}^{u} D x(t)\right) \\
& \geq x(t)\left(\frac{\beta}{2}-\left(b^{u}+e_{1}^{u} D\right) x(t)\right) .
\end{aligned}
$$

By Lemma 2.1, we have

$$
\liminf _{t \rightarrow+\infty} x(t) \geq \frac{\beta}{2\left(b^{u}+e_{1}^{u} D\right)} \triangleq m_{1}
$$

Thus, there exists $T_{2}>T_{1}+K$ such that for all $t>T_{2}$,

$$
x(t) \geq \frac{m_{1}}{2} .
$$

Inequality (2.11) together with the third equation of (1.4) leads to

$$
\dot{u}(t) \geq-d_{1}^{u} u(t)+p_{1}^{l} \frac{m_{1}}{2} \quad \text { for all } t>T_{2}+\tau .
$$

By applying Lemma 2.2(ii) to the above differential inequality, we have

$$
\liminf _{t \rightarrow+\infty} u(t) \geq \frac{p_{1}^{l} m_{1}}{2 d_{1}^{u}} \triangleq l_{1}
$$


Obviously, $m_{1}$ and $l_{1}$ are independent of the solution of system (1.4). Inequalities (2.10) and (2.12) show that the conclusion of Lemma 2.4 holds. The proof is completed.

Lemma 2.5 For any positive solution $(x(t), y(t), u(t), v(t))^{T}$ of system (1.4) with initial conditions (1.6), there exist two positive constants $m_{2}$ and $l_{2}$ satisfying

$$
\liminf _{t \rightarrow+\infty} y(t) \geq m_{2}, \quad \liminf _{t \rightarrow+\infty} v(t) \geq l_{2},
$$

where $m_{2}$ and $l_{2}$ are defined in the proof.

Proof The proof of Lemma 2.5 is similar to the proof of Lemma 2.4. However, for the sake of completeness, we give the complete proof here.

According to Lemma 2.3, there exists enough large $T_{3}>0$ such that for $t \geq T_{3}$,

$$
y(t) \leq 2 M_{2}, \quad v(t) \leq 2 L_{2} .
$$

Thus, it follows from (2.13) and the second equation of system (1.4) that

$$
\begin{aligned}
\dot{y}(t) & =y(t)\left(a_{2}(t)-\frac{r(t) y(t)}{x(t)+k(t)}-e_{2}(t) v(t)\right) \\
& \geq y(t)\left(a_{2}^{l}-\frac{2 r^{u} M_{2}}{k^{l}}-2 e_{2}^{u} L_{2}\right) \\
& \triangleq P y(t),
\end{aligned}
$$

where $P=a_{2}^{l}-\frac{2 r^{u} M_{2}}{k^{l}}-2 e_{2}^{u} L_{2}<0$.

Integrating both sides of (2.14) from $\eta(\eta \leq t)$ to $t$, leads to

$$
\frac{y(t)}{y(\eta)} \geq \exp \{P(t-\eta)\}
$$

or

$$
y(\eta) \leq y(t) \exp \{-P(t-\eta)\} .
$$

Particularly, take $\eta=t-\tau$, one can get

$$
y(t-\tau) \leq y(t) \exp \{-P \tau\}
$$

Substituting (2.16) into the fourth equation of system (1.4) leads to

$$
\dot{v}(t) \leq-d_{2}^{l} v(t)+p_{2}^{u} y(t) \exp \{-P \tau\}
$$

Applying Lemma 2.2(i) to the above differential inequality, for $0 \leq s \leq t$, one has

$$
\begin{aligned}
& v(t) \quad \\
& \quad \underset{\text { from (2.15) }}{\leq} v(t-s) \exp \left\{-d_{2}^{l} s\right\}+\int_{t-s}^{t} p_{2}^{u} y(\eta) \exp \{-P \tau\} \exp \left\{d_{2}^{l}(\eta-t)\right\} d \eta \\
& \left.\quad d_{2}^{l} s\right\}
\end{aligned}
$$




$$
\begin{gathered}
+\int_{t-s}^{t} p_{2}^{u} y(t) \exp \{-P(t-\eta)\} \exp \{-P \tau\} \exp \left\{d_{1}^{l}(\eta-t)\right\} d \eta \\
\leq \quad v(t-s) \exp \left\{-d_{2}^{l} s\right\}+p_{2}^{u} y(t) \frac{1}{P}(1-\exp \{-P s\}) \exp \{-P \tau\},
\end{gathered}
$$

where we have used the fact that $\max _{\eta \in[t-s, t]} \exp \left\{d_{2}^{l}(\eta-t)\right\}=\exp \{0\}=1$.

Note that there exists $K_{1}$ such that $2 e_{2}^{u} L_{2} \exp \left\{-d_{2}^{l} K_{1}\right\}<\frac{a_{2}^{l}}{2}$ as $s \geq K_{1}$. In fact, we can choose $K_{1}>\frac{1}{d_{2}^{l}} \ln \frac{4 e_{2}^{u} L_{2}}{a_{2}^{l}}$. And so, fix $K_{1}$, combined with (2.18), we can obtain

$$
\begin{aligned}
v(t) & \leq v\left(t-K_{1}\right) \exp \left\{-d_{2}^{l} K_{1}\right\}+p_{2}^{u} y(t) \frac{1}{P}\left(1-\exp \left\{-P K_{1}\right\}\right) \exp \{-P \tau\} \\
& \leq 2 L_{2} \exp \left\{-d_{2}^{l} K_{1}\right\}+p_{2}^{u} y(t) \frac{1}{P}\left(1-\exp \left\{-P K_{1}\right\}\right) \exp \{-P \tau\} \\
& \leq 2 L_{2} \exp \left\{-d_{2}^{l} K_{1}\right\}+B y(t)
\end{aligned}
$$

for all $t>T_{1}+K_{1}$, where $B=p_{2}^{u} \frac{1}{P}\left(1-\exp \left\{-P K_{1}\right\}\right) \exp \{-P \tau\}$.

Substituting (2.19) into the second equation of system (1.4), for all $t>T_{3}+K_{1}$, one has

$$
\begin{aligned}
\dot{y}(t) & \geq y(t)\left(a_{2}^{l}-\frac{r^{u} y(t)}{k^{l}}-e_{2}^{u}\left(2 L_{2} \exp \left\{-d_{2}^{l} K_{1}\right\}+B y(t)\right)\right) \\
& =y(t)\left(a_{2}^{l}-2 e_{2}^{u} L_{2} \exp \left\{-d_{2}^{l} K_{1}\right\}-\left(\frac{r^{u}}{k^{l}}+e_{2}^{u} B\right) y(t)\right) \\
& \geq x(t)\left(\frac{a_{2}^{l}}{2}-\left(\frac{r^{u}}{k^{l}}+e_{2}^{u} B\right) y(t)\right) .
\end{aligned}
$$

By Lemma 2.1, we have

$$
\liminf _{t \rightarrow+\infty} x(t) \geq \frac{a_{2}^{l} k^{l}}{2\left(r^{u}+e_{2}^{u} k^{l} B\right)} \triangleq m_{2}
$$

Thus, there exists $T_{4}>T_{3}+K_{1}$ such that for all $t>T_{4}$,

$$
y(t) \geq \frac{m_{2}}{2} .
$$

Inequality (2.22) together with the fourth equation of (1.4) leads to

$$
\dot{v}(t) \geq-d_{2}^{u} v(t)+p_{2}^{l} \frac{m_{2}}{2} \quad \text { for all } t>T_{4}+\tau .
$$

By applying Lemma 2.2(ii) to the above differential inequality, we have

$$
\liminf _{t \rightarrow+\infty} v(t) \geq \frac{p_{2}^{l} m_{2}}{2 d_{2}^{u}} \triangleq l_{2}
$$

Obviously, $m_{2}$ and $l_{2}$ are independent of the solution of system (1.4). Inequalities (2.21) and (2.23) show that the conclusion of Lemma 2.5 holds. The proof is completed.

Lemmas 2.3-2.5 show that the conclusion of Theorem B holds. 


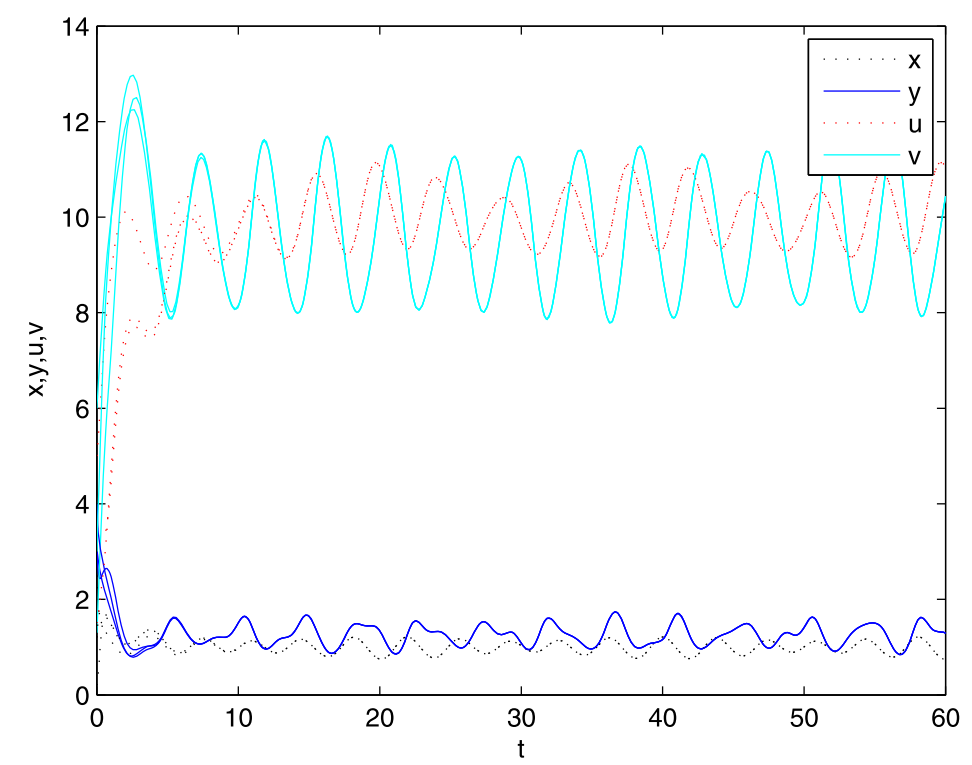

Figure 1 Dynamic behavior of system (3.1) with the initial conditions $(x(0), y(0), u(0), v(0))=(1.2,4,1.5,3)^{T},(0.2,3,3.5,1.3)^{T}$, and $(2.5,3,5,6)^{T}$, respectively.

\section{Examples and numeric simulations}

Consider the following example:

$$
\left\{\begin{array}{l}
\dot{x}(t)=x(t)\left(11+\cos \sqrt{3} t-6 x(t)-\frac{(0.8+0.7 \sin \sqrt{5} t) y(t)}{20+(12+\sin \sqrt{7} t) x(t)+5 y(t)}-0.5 u(t)\right) \\
\dot{y}(t)=y(t)\left(5+0.3 \sin \sqrt{7} t-\frac{(5+0.2 \cos \sqrt{11} t) y(t)}{x(t)+2}-0.3 v(t)\right) \\
\dot{u}(t)=-0.2 u(t)+(2+\sin \sqrt{2} t) x(t-0.5) \\
\dot{v}(t)=-(0.4+0.2 \cos \sqrt{2} t) v(t)+3 y(t-0.5)
\end{array}\right.
$$

in this case, we have

$$
a_{1}^{l}-\frac{c^{u}}{\gamma^{l}}=9.7>0
$$

Equation (3.2) shows that $\left(\mathrm{H}_{2}\right)$ holds, so system (3.1) is permanent according to Theorem B. Our numerical simulation supports our result (see Figure 1). However,

$$
a_{1}^{l}-\frac{c^{u}}{\gamma^{l}}-e_{1}^{u} L_{1}=-5.3<0 \quad \text { and } \quad a_{2}^{l}-e_{2}^{u} L_{2}=-15.175<0,
$$

that is to say, $\left(\mathrm{H}_{1}\right)$ does not hold and we cannot obtain the result of the permanence from Theorem A. Thus our results improve the main results in Zhang et al. [10].

Competing interests

The author declares that there is no conflict of interests regarding the publication of this paper.

Author's contributions

The author wrote the manuscript carefully, read and approved the final manuscript.

\section{Acknowledgements}

The author would like to thank the two anonymous referees for their constructive suggestions on improving the presentation of the paper. 
Received: 31 July 2014 Accepted: 25 February 2015 Published online: 07 March 2015

\section{References}

1. Leslie, PH: Some further notes on the use of matrices in population mathematics. Biometrika 35, 213-245 (1948)

2. Leslie, PH: A stochastic model for studying the properties of certain biological systems by numerical methods. Biometrika 45, 16-31 (1958)

3. Aziz-Alaoui, MA, Daher Okiye, M: Boundedness and global stability for a predator-prey model with modified Leslie-Gower and Holling-type II schemes. Appl. Math. Lett. 16, 1069-1075 (2003)

4. Yu, S: Global asymptotic stability of a predator-prey model with modified Leslie-Gower and Holling-type II schemes. Discrete Dyn. Nat. Soc. 2012, Article ID 208167 (2012)

5. Zhu, Y, Wang, K: Existence and global attractivity of positive periodic solutions for a predator-prey model with modified Leslie-Gower Holling-type II schemes. J. Math. Anal. Appl. 384, 400-408 (2011)

6. Yu, S, Chen, F: Almost periodic solution of a modified Leslie-Gower predator-prey model with Holling-type II schemes and mutual interference. Int. J. Biomath. 7, 1450028 (2014)

7. Yu, S: Global stability of a modified Leslie-Gower model with Beddington-DeAngelis functional response. Adv. Differ. Equ. 2014, 84 (2014)

8. Pal, P, Mandal, P: Bifurcation analysis of a modified Leslie-Gower predator-prey model with Beddington-DeAngelis functional response and strong Allee effect. Math. Comput. Simul. 97, 123-146 (2014)

9. Zhang, Z: Almost periodic solution of a modified Leslie-Gower predator-prey model with Beddington-DeAngelis functional response. J. Appl. Math. 2013, Article ID 834047 (2013)

10. Zhang, K, Li, J, Yu, A: Almost periodic solution of a modified Leslie-Gower predator-prey model with Beddington-DeAngelis functional response and feedback controls. Abstr. Appl. Anal. 2014, Article ID 252579 (2014)

11. Chen, F, Yang, J, Chen, L: Note on the persistent property of a feedback control system with delays. Nonlinear Anal., Real World Appl. 11, 1061-1066 (2010)

12. Chen, F, Yang, J, Chen, L, Xie, X: On a mutualism model with feedback controls. Appl. Math. Comput. 214, 581-587 (2009)

13. $\mathrm{Wu}, \mathrm{H}, \mathrm{Yu}, \mathrm{S}$ : Permanence, extinction, and almost periodic solution of a Nicholson's blowflies model with feedback control and time delay. Discrete Dyn. Nat. Soc. 2013, Article ID 798961 (2013)

14. Chen, F, Li, Z, Huang, Y: Note on the permanence of a competitive system with infinite delay and feedback controls. Nonlinear Anal., Real World Appl. 8, 680-687 (2007)

\section{Submit your manuscript to a SpringerOpen ${ }^{\circ}$ journal and benefit from:}

- Convenient online submission

Rigorous peer review

- Immediate publication on acceptance

- Open access: articles freely available online

- High visibility within the field

- Retaining the copyright to your article 